\title{
Rétro-BIM ou la question de l'intégration : une revue anachronique
}

\section{Integration in BIM: An anachronistic literature review}

\author{
Léa Sattler ${ }^{*}$, Samir Lamouri² et Robert Pellerin ${ }^{3}$ \\ ${ }^{1}$ Architecte - Doctorante ENSAM École Nationale Supérieure des Arts et Métiers \\ ${ }^{2}$ Professeur des universités - ENSAM \\ ${ }^{3}$ Professeur - Polytechnique Montréal
}

\begin{abstract}
Résumé. Si la collaboration digitale autour du BIM est toujours un défi de nos jours, comment cette problématique était-elle traitée dans la période de gestation du BIM, avant les années 2000 ? Le présent article mène une revue de littérature rétroactive sur le sujet, à travers 6 thèmes récurrents (la comparaison avec les industries digitalisées, la conjonction de l'organisationnel et du numérique, le bouleversement des temporalités du projet, les nouveaux modèles de données, les processus dynamiques et l'anticipation), dans le but de donner une perspective historique à cette question actuelle.
\end{abstract}

Mots-clés. BIM, Intégration, Organisation, Interopérabilité

\begin{abstract}
If BIM digital collaboration is still a challenge today, how was this issue addressed in the BIM gestation period before the 2000s? This article retrospectively reviews the scientific literature on the subject, through 6 recurring themes (comparison with digitalized industries, the conjunction of organizational and digital modes, the disruption of project temporalities, new data models, dynamic processes and anticipation), in order to give a historical perspective to this current issue.
\end{abstract}

Keywords. BIM, Integration, Organization, Interoperability

\section{Introduction}

Si la pratique du BIM (Building Information Modeling) s'est imposée depuis une vingtaine d'années dans l'industrie du bâtiment, il semble pourtant qu'une des promesses de cette transition digitale n'ait pas été tenue : celle d'une collaboration facilité, plus fluide et plus précise (Eastman, Eastman, Teicholz, \& Sacks, 2011). En effet, la collaboration BIM n'aurait pas encore atteint un niveau satisfaisant, et deux facteurs sont régulièrement

\footnotetext{
* Corresponding author: lea.sattler@ensam.eu
} 
invoqués pour expliquer ce phénomène : d'une part, le manque d'interopérabilité technique des maquettes numériques (Hu, Zhang, Wang, \& Kassem, 2016; McGraw - Hill, 2012), et d'autre part, le manque de réflexion sur une réforme des modes opératoires organisationnels, contractuels et économiques dans cette industrie fragmentée et basée sur la production massive de prototypes (Arayici, Fernando, Munoz, \& Bassanino, 2018; Grilo, Zutshi, Jardim-Goncalves, \& Steiger-Garcao, 2013).

Dans un précédent travail (Sattler, Lamouri, Pellerin, Paviot, \& Deneux, 2019), nous nous sommes attachés à remonter cette double piste de la collaboration humaine et digitale dans le BIM - question devenue brûlante récemment (Santos, Costa, \& Grilo, 2017), dont nous avons dressé un état de l'art sur une période récente s'étendant de 2013 à 2018. L'un des résultats de cette investigation est le constat selon lequel ces deux aspects de la question ne sont que trop rarement traités de manière concourante: il y a une division dans l'approche même du sujet, avec certains travaux exclusivement dédiés à l'interopérabilité technique, et d'autres à la collaboration (aspect communicationnel, organisationnel, contractuel, économique).

Une plongée dans le passé pouvant parfois éclairer de manière surprenante le présent (et le futur), nous nous sommes demandé ce que donnerait cette même question au sujet de la collaboration et de l'interopérabilité BIM en considérant une période différente. La période de gestation du BIM - les années 90 - nous a semblé être un terrain particulièrement fertile. Ainsi : quid de la collaboration digitale dans le bâtiment, à une époque où le BIM était encore en gestation? Comment ce regard rétroactif peut-il éclairer les problématiques actuelles?

Pour répondre à cette question, nous avons mené une brève et restreinte revue de littérature, dont nous détaillerons la méthodologie dans la partie 2. La partie 3 présentera l'analyse, et la partie 4 une conclusion.

\section{Méthodologie}

Nous détaillerons ici la manière dont nous avons constitué notre corpus de littérature scientifique.

Nous avons rencontré un premier obstacle méthodologique: notre question frise l'anachronisme. En effet, avant 2000, le BIM tel que nous le connaissons aujourd'hui n'existait pas : cette méthodologie de conduite de projet s'est développée dans le cercle des grands projets complexes de la $1^{\text {re }}$ décennie des années 2000 , et démocratisée dans la décennie suivante (Sattler \& Maigne, 2018). Le BIM était toutefois en gestation dans les années 90 : une des premières mentions scientifiques du terme «building information model» se trouve dans un article sur la modélisation multi-vues paru en 1992 (van Nederveen \& Tolman, 1992), considéré comme un des premiers articles sur le BIM. En cette période de «premier tournant digital» de l'industrie du bâtiment (Carpo, 2017), des architectes pionniers comme Frank O. Gehry et Norman Foster s'emparaient des suites de logiciels orientées PDM-PLM («Product Data Management - Product Lifecycle Management») afin de créer des modélisations de données géométriques et non géométriques de certains de leurs bâtiments à morphologie complexe, et ce alors qu'aucun modèle de données commun n'existait dans l'industrie du bâtiment - l'IFC étant apparu en 1997.

Ainsi l'acronyme «BIM» avait une prégnance trop faible avant 2000, et pour évoquer la «collaboration BIM», nous avons utilisé son synonyme de l'époque: le mot «intégration ». L'intégration désigne le processus qui consiste à articuler différents soussystèmes en un système centralisé - en d'autres termes, il s'agit de transformer un système 
fragmenté en un système symbiotique (Burbidge, Falster, Riis, \& Svendsen, 1987). Comme le BIM, l'intégration relève à la fois d'un pendant organisationnel et digital.

Afin de collecter des articles, nous avons lancé deux groupes de mots-clefs dans la base de données SCOPUS, afférents à l'industrie du bâtiment en phase conception et construction ( AEC, Building Industry, Design and Construction») et les différentes déclinaisons du mot intégration («Integration, Integrated, Integrate»). Nous avons limité notre recherche aux articles publiés avant l'an 2000, que nous situons donc comme la date symbolique de naissance du BIM. Enfin, nous avons limité l'occurrence des mots cibles aux titres des articles, afin d'obtenir un échantillon certes restreint, mais pertinent et resserré. Notons que la présente recherche peut être considérée comme un prééchantillonnage, préalable à une étude plus vaste de la littérature scientifique à mener dans de futurs travaux. La chaîne de recherche est la suivante :

TITLE ( ( aec OR "building industry" OR "design and construction" ) AND
(integration OR integrated OR integrate) ) AND PUBYEAR < 2000 AND ( LIMIT-
TO ( SUBJAREA , "ENGI" ) OR LIMIT-TO ( SUBJAREA , "COMP" )) AND
(LIMIT-TO ( DOCTYPE, "ar" ) ) AND ( LIMIT-TO ( LANGUAGE, "English" ))

Cette recherche a donné 19 articles, dont nous avons manuellement exclu 6 articles qui n'étaient pas afférents à l'industrie du bâtiment. Une lecture soignée de chaque article a fait émerger 6 thèmes récurrents, par les prismes desquels nous allons analyser le présent corpus.

\section{Analyse}

\subsection{Comparaison aux industries digitalisées}

Il est courant d'évoquer le BIM comme un descendant du PLM, méthode de conception et gestion digitale de produit implémentée dans les industries manufacturières, automobiles et aéronautiques. Que nous dit ce corpus quant à ce référentiel pour l'industrie du bâtiment?

Dans un article de 1987 (Howard, Levitt, Paulson, Pohl, \& Tatum, 1989), Howard commence avec ce constat : l'industrie du bâtiment est très fragmentée, et c'est une des causes de son retard en termes de digitalisation. Beaucoup serait à apprendre des industries manufacturières ou automobiles. Une emphase est mise sur un besoin urgent de gagner en productivité, productivité qui baisse dans le bâtiment, mais qui augmente dans les autres industries (Reinschmidt, Griffis, \& Bronner, 1991). Plusieurs articles (Alfares, M., Seireg, A., 1996; Yamazaki, 1992) évoquent directement la possibilité d'adapter le CIM («Computer Integrated Manufacturing») en CIC («Computer Integrated Construction»). Au-delà du CIM, la notion d'ingénierie concourante (Evbuomwan \& Anumba, 1998), alors implantée dans l'industrie manufacturière, semble aussi être candidate pour une application dans le bâtiment. Par ailleurs, le terme de «product-modelling », venant du PLM, est dérivé en «project-modelling » (Luiten, Tolman, \& Fischer, 1998) : une appropriation directe de ce concept par l'industrie du bâtiment.

Si les précédents articles soulignent généralement la fragmentation comme élément différenciant l'industrie du bâtiment des autres industries, Eastman (Eastman, Chase, \& Assal, 1993) insiste sur l'aspect holistique du projet de construction, basé sur une multiplicité de disciplines interconnectées, et propose d'approcher le sujet de l'intégration dans la construction via l'idée de matrice. Autre prise de distance vis-à-vis des autres industries : le bâtiment serait en retard en termes de digitalisation justement parce qu'on 
aurait voulu calquer ses déploiements digitaux sur le modèle du PLM (El-Bibany \& Paulson, 1994), sans analyser sa capacité de se conformer à ce modèle. Par exemple, l'industrie du bâtiment n'est pas assez centralisée (Norton, Lo, Cronin, \& Yohanis, 1995), ce qui n'est pas le cas de l'industrie manufacturière. Similairement, d'après Brown (Brown et al., 1995), le product-modeling n'est pas adapté au bâtiment, avec sa grande variété de métiers et de niveaux de détail. D'après lui, les développements digitaux dans l'AEC devraient être pensés via une approche globale, à l'échelle de l'industrie, et non via une approche locale par projet.

Ainsi, la question de l'intégration dans le bâtiment, originellement liée à une comparaison avec les industries plus digitalisées, se libère progressivement de ce référentiel, et est traitée sous l'angle de ses spécificités: une industrie décentralisée, incluant une importante variété de métiers.

\subsection{Conjonction de l'aspect organisationnel et technologique}

Comme mentionné plus haut, la conjonction des aspects organisationnels et technologiques fait défaut aujourd'hui dans le traitement du sujet de la collaboration BIM. Ce constat était-il valable à l'époque de la gestation du BIM ?

Howard (Howard et al., 1989) décrit le paradoxe suivant : l'introduction des ordinateurs dans l'industrie du bâtiment a modifié les manières de générer des documents à l'intérieur de certaines organisations, mais pas les méthodes d'échanges de données à l'extérieur, audelà des frontières organisationnelles, c'est-à-dire entre plusieurs entreprises. Il donne l'exemple de deux bureaux d'études, dont l'un produit des documents CAD («Computer Integrated Design »), qui sont «dé-CADés » par le second via une impression papier, qui devra être re-digitalisée et éventuellement «re-CADée» par le premier lors d'une future itération. Paradoxalement donc, la digitalisation dans le bâtiment crée une couche supplémentaire de fragmentation, entre les digitalisés et les autres - ce qui ne va pas exactement dans le sens de l'intégration. Trente ans plus tard, ce constat est toujours valable au sujet du BIM : les entreprises qui n'utilisent pas de maquettes numériques extraient des données 2D et produisent hors du circuit BIM. Howard émet donc l'idée que l'intégration dans le bâtiment devrait induire de nouvelles dispositions économiques et organisationnelles dans cette industrie, insufflées par les voies industrielles, mais aussi académiques.

Cette nécessité de modification structurelle de l'industrie du bâtiment pour supporter la digitalisation est actée dans la littérature (Fenves, Flemming, Hendrickson, Maher, \& Schmitt, 1990; Reinschmidt et al., 1991). Si la visée est bien, in fine, un système de production «totale», où le travail manuel serait réduit et beaucoup de tâches automatisées (Alfares, M., Seireg, A., 1996), il est préconisé d'appliquer l'intégration aux phases de conception et d'ingénierie, avant de l'étendre à la robotique, alors peu implantée dans le bâtiment (Yamazaki, 1992). Norton (Norton et al., 1995) évoque l'importance de se pencher sur les interfaces et dialogues homme-machine, et de mettre en place de nouveaux processus et cadres de travail. D'après El-Bibany (El-Bibany \& Paulson, 1994), une distinction s'impose entre intégrations logicielles et la collaboration humano-digitale («human-computer-based collaboration»). D'après lui, les précédentes recherches se concentrent sur le $1^{\text {er }}$ volet et pas assez sur le $2^{\text {nd }}$ : la question de la mise en œuvre d'une intégration technique dans l' « organisation dynamique » de l'industrie du bâtiment reste à résoudre.

Kiwan (Kiwan \& Munns, 1996) souligne l'importance de créer une adhésion des utilisateurs aux systèmes digitaux. Une solution proposée est de calquer les processus digitaux sur les processus existants, à savoir les processus papier, afin de réduire la courbe 
d'apprentissage pour les utilisateurs. Il semble que cette préconisation ait été suivie, de fait ou à dessein, si on considère que les workflows BIM sont toujours basés sur les processus $2 \mathrm{D}$, et que les plans et coupes restent les seuls documents contractuels utilisés pour la construction en 2019 en France. Plus ambitieux, Luiten (Luiten et al., 1998), conseille de faire découler les processus digitaux des activités cognitives à l'œuvre dans la conception et la construction - soit de s'appuyer sur la manière dont les professionnels «parlent et pensent». Il évoque aussi un plan progressif de déploiement de processus collaboratifs digitaux : à l'intérieur d'une société, puis entre deux sociétés partenaires, puis entre plusieurs sociétés. Il est intéressant de noter que 20 ans plus tard, la collaboration BIM fonctionne essentiellement dans le $1^{\text {er }}$ cas de figure : la collaboration inter-équipes et intermétiers est toujours fastidieuse, traitée de manière ad-hoc, et basée sur l'échange de silos de données (Sattler et al., 2019). L'implémentation de l'ingénierie concourante change, elle, radicalement la donne, et impose d'adopter des nouveaux processus économiques et organisationnels pour soutenir l'intégration (Evbuomwan \& Anumba, 1998).

Il semble clair que dans les années 90 , la mise en œuvre des processus collaboratifs digitaux n'était pas envisagée sans une évolution importante et conjointe des modalités de fonctionnement de l'industrie du bâtiment. Or, cette conjonction des aspects technologiques et organisationnels est justement ce qui fait défaut aux réflexions similaires de la fin des années 2010 (Sattler et al., 2019).

\subsection{Temporalité}

Associée à une hausse de productivité, la pratique du BIM est souvent la promesse d'une accélération du processus de projet - promesse non forcément tenue en pratique. L'analyse des temporalités évoquées dans notre corpus suggère 3 catégories de temporalités associées à l'intégration.

Le $1^{\text {er }}$ besoin, c'est de gagner du temps (Fenves et al., 1990) : réduire les temps de construction (Howard et al., 1989), augmenter les vitesses d'assemblage, accélérer les cadences et la productivité (Eastman et al., 1993), supprimer tout délai, tout devant arriver «juste à temps » (Alfares, M., Seireg, A., 1996) - en un moment, ne pas «gâcher » de temps (Brown et al., 1995). C'est la $1^{\text {ère }}$ promesse de l'intégration : améliorer la productivité.

Le $2^{\text {nd }}$ besoin, lié au $1^{\text {er }}$, c'est que tout arrive plus tôt (Alshawi \& Underwood, 1996; Norton et al., 1995; Yamazaki, 1992) : l'idée est de procéder, en amont du projet, à des analyses et à des développements qui auparavant se faisaient en aval du projet. C'est la base de l'ingénierie concourante, qui ne peut se faire sans processus intégratif. Grâce à cette capacité d'intégrer dès la conception des paramètres qui auparavant n'étaient appréhendables que plus tardivement, beaucoup de décisions de conception sont prises plus tôt.

Le $3^{\text {ème }}$ besoin, en conséquence, c'est le temps réel (Howard et al., 1989), l'idée qu'à tout moment le processus de design peut-être impacté par un nouveau paramètre et que le processus de simulation peut être relancé (El-Bibany \& Paulson, 1994; Luiten et al., 1998) : c'est le temps simultané (Evbuomwan \& Anumba, 1998). Paradoxalement, c'est aussi le temps du retour en arrière: on peut toujours revenir une étape en amont, changer un paramètre et relancer un calcul. C'est enfin le temps continu qui sera évoqué des décennies plus tard au sujet l'industrie 4.0. D'après Luiten (Luiten et al., 1998), l'intégration devrait transformer le processus de construction en un «processus continu d'échange d'information, à travers lequel tous les acteurs seront impliqués à tout moment ».

En conclusion, il semble que dès les années 90 , la promesse du temps réel et de la continuité temporelle ait commencé à s'immiscer dans les considérations technologiques. 
En pratique, si on s'en est rapprochés, il semble que nous sommes aujourd'hui bien loin d'une telle réalité : les projets BIM suivent toujours des enchaînements de phases topdown, par à-coups, et les différentes équipes ne collaborent pas en temps réel, mais via des soumissions hebdomadaires ou mensuelles de silos de données (Ciribini, Mastrolembo Ventura, \& Paneroni, 2016).

\subsection{Modèles de données}

Au fil de notre corpus, un questionnement émerge - celui des modèles de données pour l'industrie du bâtiment.

Fenves (Fenves et al., 1990) propose un système intégré impliquant 7 logiciels travaillant ensemble. Il se concentre sur l'idée d'articulation entre plusieurs systèmes, érigeant l'échange de données en atome du processus intégratif. Cette approche sera plus tard décriée par Norton (Norton et al., 1995), car jugée trop mécaniste et rigide. De plus, Norton souligne un manque crucial dans l'industrie du bâtiment : celui d'un domaine de données commun - manque également souligné par Kiwan (Kiwan \& Munns, 1996).

Un des freins évoqués à ce manque de modèle de données, outre le faible degré de standardisation de l'industrie du bâtiment, c'est la nature même de l'activité de conception, qui serait «à la fois la définition d'un domaine de discours, et l'exploration de ce domaine » (Eastman et al., 1993). Autrement dit, l'unicité de chaque bâtiment mène de fait à explorer des pistes de conception ad-hoc. Il est donc nécessaire d'après Eastman d'utiliser et construire des modules CAD modulaires et personnalisables. El-Bibany (El-Bibany \& Paulson, 1994) évoque lui aussi la haute incertitude des modes de collaboration dans l'industrie du bâtiment, ainsi que celle du projet lui-même. Brown (Brown et al., 1995) rappelle que l'activité de conception est «marquée par la constante génération de nouvelles tâches cibles et de nouvelles contraintes liées à ces tâches ». Ainsi l'incertitude structurelle inhérente à tout projet de construction peut-elle compliquer la tâche de trouver un modèle de données commun à l'industrie du bâtiment.

Toutefois, un modèle émerge et s'impose dans la $2^{\text {nde }}$ partie des années 90 : celui du modèle de données orienté objet (Brown et al., 1995; Kiwan \& Munns, 1996; Alshawi \& Underwood, 1996; Alfares, M., Seireg, A., 1996). Ce modèle permet d'envisager la maquette numérique comme un ensemble d'objets, ayant à la fois une représentation et un comportement, et pouvant donc encapsuler des règles métier. La modularité des composants (ou objets) ainsi que leur capacité de customisation répond aux exigences mentionnées par Eastman (Eastman et al., 1993).

Ce consensus a fait son chemin depuis dans l'industrie du bâtiment : la majorité des logiciels de modélisation BIM (comme Revit, ou Tekla) utilisés aujourd'hui sont orientés objet, de même que les logiciels de programmation visuelle comme Grasshopper (qui permet de définir des objets). Toutefois, il semble que cette recherche de modèles de données ait occulté la nécessité de traiter la question des échanges de données, et on voit aujourd'hui ressurgir dans la littérature BIM l'approche de Fenves (Fenves et al., 1990), avec des approches multi-modèles et multi-disciplinaires (Díaz, Alarcón, Mourgues, \& García, 2017; Fuchs \& Scherer, 2017; Perisic, Lazic, \& Perisic, 2016)

\subsection{Processus dynamiques}

On retrouve tout au long du corpus un invariant : la question des liens dynamiques qui composent et environnent la maquette numérique.

Le $1^{\text {er }}$ type de lien, qui peut sembler aller de soi de nos jours, ce qui n'était pas le cas dans les années 90, c'est le lien entre l'utilisateur et la maquette numérique, soit la notion 
d'interactivité, aujourd'hui désuète. Plusieurs articles ont recours à ce terme: Fenves (Fenves et al., 1990) parle de représentation graphique interactive ; Norton (Norton et al., 1995), en évoquant une interface homme-machine interactive, rappelle que «l'intervention de l'utilisateur est un ajout souhaitable au système [intégré] », comme si la communication homme-machine n'allait pas de soi. Cela nous rappelle un temps où les interfaces digitales étaient peu maniables.

Le $2^{\text {nd }}$ type de lien, c'est un lien entre les objets digitaux eux-mêmes, qu'on retrouve avec l'utilisation des bases de données relationnelles (Fenves et al., 1990; Reinschmidt et al., 1991), de systèmes de données hiérarchisés (Yamazaki, 1992), établissant des réseaux d'objets liés entre eux, des subtypes/supertypes et systèmes d'héritage (Alshawi \& Underwood, 1996). Ceci préfigure les structures de données orientées-objet mentionnées plus haut, et en particulier la naissance du format IFC. Par ailleurs, ces liens peuvent être définis par un modèle de données préalable, mais aussi par l'utilisateur, qui va créer son système de lien, pour répondre à l'aspect ad-hoc de chaque projet. (Norton et al., 1995).

Le $3^{\text {ème }}$ type de lien est similaire au second, mais avec une notion d'automation plus forte. Les objets deviennent autonomes, et échangent entre eux via des règles et «systèmes experts». Ainsi, Eastman (Eastman et al., 1993) envisage que chaque module d'une maquette communique ses «interdépendances » avec tous les autres modules, ce qui 30 ans plus tard dans le monde du BIM, est encore un vœu pieux. El-Bibany (El-Bibany \& Paulson, 1994) propose une «représentation unifiée basée sur la contrainte », c'est-à-dire une gestion des objets par des sets de contraintes paramétriques - «dépendances dynamiques de données ». La visée est la propagation automatique de modifications à tous les objets concernés, basées sur des échanges et comportements dynamiques entre les différents modules de données (Brown et al., 1995; Kiwan \& Munns, 1996). Ici est pensée une sorte de paramétricité totale entre objets, multi-métiers, multi-phases.

Cette vision d'une intégration mettant en place un système de liens dynamiques très forts domine dans le corpus - à la fois via une incorporation totale des utilisateurs au système digital, et via une autonomie de ce système. La notion d'objets interconnectés propageant des informations via leurs dépendances à travers les différentes équipes et différentes phases d'un projet n'est toujours pas réalisée aujourd'hui, ni en contexte intramétier, ni en contexte inter-métiers. On notera aussi l'évolution de ces termes : il semble que la notion d'interopérabilité, massivement convoquée par la littérature scientifique des années 2010 (Sattler et al., 2019), correspondrait à ce qui dans les années 90 était appelé interactivité. On notera aussi que ces trois mots de collaboration, interactivité et interopérabilité, ont des significations étymologiques très proches, et évoquent tous peu ou prou l'idée de «travailler/agir ensemble».

\subsection{Anticipations}

Notre corpus traitant de l'intégration dans l'industrie du bâtiment, à une époque où sa digitalisation était encore balbutiante, on retrouve au long des articles beaucoup de projections dans le futur. Que nous disent-elles vis-à-vis des développements actuels ?

Howard (Howard et al., 1989) liste les technologies à déployer pour l'intégration dans le bâtiment. La $1^{\text {ère }}$ selon lui est celle des bases de données graphiques et non graphiques ; en $2^{\text {nde }}$ place vient l'intelligence artificielle, puis l'automation et la robotique. Ces 3 dernières technologies sont, au moment de la rédaction de cet article, encore très peu déployée dans le bâtiment, et la $1^{\text {ère }}$, encore largement sous-exploitée. Howard anticipe les «bases de données de l'an $2000 »$ qui pourraient conserver toute trace de toute version d'un projet, proposer un système de référencement spatial pour l'automatisation du contrôle des machines de construction, et représenter un modèle as-built parfait. Considérant qu'en 
2019, le versionnement complet d'un projet à un niveau de granularité élevé est rarement implémenté, que les chantiers fonctionnent encore largement avec de la main-d'œuvre manuelle, et que faire le pont entre des nuages de points issus de scans $3 \mathrm{D}$ et des maquettes numériques orientées objet est encore une bravade, on peut penser qu'Howard a été un peu optimisme. Sur le même ton, Fenves (Fenves et al., 1990) évoque des «experts de substitution » qui viennent agir entre différents logiciels. L'intelligence artificielle, prenant en charge une partie du travail de l'ingénieur, est ici portée au niveau des flux de travail collectif : au niveau des échanges de données. On a donc ici l'idée d'automation de l'intégralité du processus de conception. Dans la mesure où le rule-checking automatisé est peu mis au point dans le bâtiment, et les workflows encore envisagés de manière ad-hoc et manuelle, il semble qu'il y ait encore du chemin à parcourir.

Eastman (Eastman et al., 1993) rêve de l'avènement de logiciels commerciaux dotés de modules intelligents, avec implémentation automatisée de règles métiers à différents niveaux : au niveau du corps de métier (structure, plomberies, etc.), et au niveau du programme de bâtiment (hôpitaux, écoles, etc.). À la lumière de la pratique du BIM en 2019, il semble que ces prédictions se soient partiellement réalisées : comme mentionné plus haut, la plupart des logiciels BIM sont orientés objet, et proposent des «modules intelligents» dans des bibliothèques de composants BIM. Toutefois, l'intégration automatisée de règles métiers ne va pas encore de soi. Tout d'abord, à un niveau de granularité assez bas, comme celui d'un composant de structure par exemple, les objets sont rarement complètement personnalisables, ce qui est source de frustrations : ils peuvent alors être vus comme des obstacles plutôt que comme des outils. Cela est vrai pour les logiciels BIM largement répandus, à l'exception des suites logicielles issues du PLM, qui ont une grande capacité de customisation (Sattler \& Maigne, 2018). Ensuite, l'implémentation à un niveau élevé dans la conception (comme la partition d'espace) est encore balbutiante (Morel \& Girard, 2017): elle se fait dans certaines agences d'architectures utilisant des outils de programmation comme Grasshopper, mais la partie de la conception qui consiste à analyser des règles (liées au programme, ou à la réglementation urbaine) et à les mettre en œuvre est encore majoritairement manuelle. Ceci est en passe de changer dans les années à venir, en témoignent des outils populaires comme TestFit.io, qui mettent en place des routines de partitionnement d'espace automatisées, qui, même si elles sont encore sommaires, montrent qu'une voie vers l'automation de ces tâches est ouverte.

On retrouve d'autres considérations qu'aujourd'hui encore on qualifierait de futuristes. Alfares (Alfares, M., Seireg, A., 1996) investigue la possibilité d'automatiser totalement la construction d'un bâtiment en béton armé. Il décrit un cadre où les robots reçoivent des «instructions complètes» depuis une base de données, à travers laquelle toutes les informations transitent. En 2019, on est encore loin de ce stade - le travail sur le chantier reste à majorité manuel, et les robots sont rarement présents. Quant à Luiten (Luiten et al., 1998), il expose les prérequis pour l'implémentation de systèmes intégratifs dans le domaine de la conception et construction. Ainsi l'infrastructure logicielle idéale ne devrait requérir aucune interaction humaine pour interpréter ou transférer la donnée.

Les anticipations du corpus nous montrent qu'un rêve d'automation plus complet était à l'œuvre dans les années 90, touchant toutes les couches de l'industrie du bâtiment: la conception architecturale, l'ingénierie, la construction.

\section{Conclusion}

Dans le présent article, nous avons effectué un saut dans le passé, et mené une revue de littérature au sujet de la question de la collaboration digitale dans l'industrie du bâtiment dans la période pré-BIM, soit antérieure aux années 2000. Le BIM étant encore en gestation 
à cette époque, nous sommes passés par la notion d'intégration, qui préfigurait ce qui allait devenir plus tard le BIM. Nous avons analysé soigneusement un corpus restreint de la littérature scientifique de l'époque, via 6 thèmes récurrents : la comparaison aux industries déjà digitalisées, la conjonction entre une intégration organisationnelle et digitale, les temporalités envisagées, la recherche d'un modèle de données commun, l'aspect dynamique des processus et workflows, et les anticipations sur un futur du BIM.

Parmi les promesses technologiques formulées dans les années 90, nous avons pu identifier celles qui se sont réalisées - et celles dont la tenue reste à venir. Le développement d'un modèle de données commun était très manifestement une gageure importante à réaliser à l'époque. Même si l'IFC, format neutre de facto de l'industrie du bâtiment, est aujourd'hui considéré comme imparfait par les industriels, il n'en est pas moins une base commune utilisée actuellement dans la grande majorité des projets BIM, avec laquelle tous les logiciels sont compatibles (même si la qualité de cette compatibilité fait parfois débat). Même constat pour la modélisation orientée-objet, qui est la règle pour les logiciels BIM, désormais largement répandus. Étant donné que l'industrie du bâtiment partait de zéro sur ces sujets, un long chemin a été parcouru, et a partiellement porté ses fruits. La prise de distance vis-à-vis des logiques PLM a sans doute été une étape importante pour développer ce modèle de données commun; mais un retour à ce référentiel pour traiter les échanges de données de manière globale semble souhaitable.

Par ailleurs, certaines anticipations de l'époque de la gestation du BIM nous semblent encore futuristes aujourd'hui : le temps réel et continu n'est toujours pas la cadence par défaut des projets de bâtiment, pas plus qu'une interconnexion fine entre les données BIM à travers les différentes équipes n'est mise en place. L'intelligence artificielle est peu employée, si ce n'est dans quelques programmes de recherche de pointe, et les chantiers ne sont ni automatisés ni robotisés. Il semble qu'une vision plus holistique de l'intégration dans le bâtiment était à l'œuvre dans les années 90 : à la fois dans la vision technologique d'une sorte de paramétricité et interdépendance totale des différentes données, et dans l'articulation de cette vision technologique avec une réforme des dispositions organisationnelles, contractuelles et économiques de l'industrie du bâtiment. Ce rappel d'une intrication de ces deux aspects, technologique et organisationnel, nous semble crucial aujourd'hui, dans la mesure où le développement du BIM se heurte encore régulièrement aux modes opératoires traditionnels toujours à l'œuvre dans le bâtiment - modes qui, malgré la digitalisation, n'ont pas encore été réellement remis en cause.

Cette revue de littérature a été menée sur un échantillon de littérature restreint, qui pourrait servir de base à un état de l'art plus vaste, à mener dans de futurs travaux.

\section{Bibliographie}

Alfares, M., Seireg, A. (1996). An integrated system for computer-aided design and constructionof reinforced concrete buildings using modular forms. Automation in Construction.

Alshawi, M., \& Underwood, J. (1996). Applying object-oriented analysis to the integration of design and construction. Automation in Construction, 5(2), 105-121. https://doi.org/10.1016/0926-5805(95)00023-2

Arayici, Y., Fernando, T., Munoz, V., \& Bassanino, M. (2018). Interoperability specification development for integrated BIM use in performance based design. Automation in Construction, 85, 167-181. https://doi.org/10.1016/j.autcon.2017.10.018

Brown, F. E., Cooper, G. S., Ford, S., Aouad, G., Brandon, P., Child, T., ... Young, B. (1995). An integrated approach to CAD: modelling concepts in building design and 
construction. Design Studies, 16(3), 327-347. https://doi.org/10.1016/0142694X(94)00002-U

Burbidge, J. L., Falster, P., Riis, J. O., \& Svendsen, O. M. (1987). Integration in Manufacturing. Computers in Industry, 9(4), 297-305. https://doi.org/10.1016/01663615(87)90103-5

Carpo, M. (2017). The Second Digital Turn, Design Beyond Intelligence. MIT Press.

Ciribini, A. L. C., Mastrolembo Ventura, S., \& Paneroni, M. (2016). Implementation of an interoperable process to optimise design and construction phases of a residential building: A BIM Pilot Project. Automation in Construction, 71, 62-73. https://doi.org/10.1016/j.autcon.2016.03.005

Díaz, H., Alarcón, L. F., Mourgues, C., \& García, S. (2017). Multidisciplinary Design Optimization through process integration in the AEC industry: Strategies and challenges. Automation in Construction, 73, 102-119. https://doi.org/10.1016/j.autcon.2016.09.007

Eastman, C. M., Chase, S. C., \& Assal, H. H. (1993). System architecture for computer integration of design and construction knowledge. 13.

Eastman, C. M., Eastman, C., Teicholz, P., \& Sacks, R. (2011). BIM Handbook: A Guide to Building Information Modeling for Owners, Managers, Designers, Engineers and Contractors. Hoboken, NJ: John Wiley \& Sons.

El-Bibany, H., \& Paulson, B. C. (1994). Collaborative Knowledge-integration Systems: A Tool for AEC Design, Management, and Coordination. Computer-Aided Civil and Infrastructure Engineering, 9(1), 29-40. https://doi.org/10.1111/j.14678667.1994.tb00359.x

Evbuomwan, N. F. O., \& Anumba, C. J. (1998). An integrated framework for concurrent life-cycle design and construction. Advances in Engineering Software, 29(7-9), 587-597. https://doi.org/10.1016/S0965-9978(98)00024-6

Fenves, S. J., Flemming, U., Hendrickson, C., Maher, M. L., \& Schmitt, G. (1990). Integrated software environment for building design and construction. Computer-Aided Design, 22(1), 27-36. https://doi.org/10.1016/0010-4485(90)90026-9

Fuchs, S., \& Scherer, R. J. (2017). Multimodels - Instant nD-modeling using original data. Automation in Construction, 75, 22-32. https://doi.org/10.1016/j.autcon.2016.11.013

Grilo, A., Zutshi, A., Jardim-Goncalves, R., \& Steiger-Garcao, A. (2013). Construction collaborative networks: the case study of a building information modelling-based office building project. International Journal of Computer Integrated Manufacturing, 26(1-2), 152-165. https://doi.org/10.1080/0951192X.2012.681918

Howard, H. C., Levitt, R. E., Paulson, B. C., Pohl, J. G., \& Tatum, C. B. (1989). Computer Integration: Reducing Fragmentation in AEC Industry. Journal of Computing in Civil Engineering, 3(1), 18-32. https://doi.org/10.1061/(ASCE)0887-3801(1989)3:1(18)

Hu, Z.-Z., Zhang, X.-Y., Wang, H.-W., \& Kassem, M. (2016). Improving interoperability between architectural and structural design models: An industry foundation classes-based approach with web-based tools. Automation in Construction, 66, 29-42. https://doi.org/10.1016/j.autcon.2016.02.001

Kiwan, M. S., \& Munns, A. K. (1996). A neutral object data model for integrated building design and construction environment. Advances in Engineering Software, 25(2-3), 131-140. https://doi.org/10.1016/0965-9978(95)00105-0

Luiten, G. T., Tolman, F. P., \& Fischer, M. A. (1998). Project-modelling in AEC to integrate design and construction. Computers in Industry, 35(1), 13-29. https://doi.org/10.1016/S0166-3615(97)00081-X

McGraw - Hill. (2012). The Business Value in BIM North America - Multi-year Trend Analysis and Users Ratings (2007-2012). Retrieved from McGraw - Hill website: 
https://bimforum.org/wp-content/uploads/2012/12/MHC-Business-Value-of-BIM-in-NorthAmerica-2007-2012-SMR.pdf

Morel, P., \& Girard, C. (2017). Nous pensons qu'il faut dynamiter la façon d'enseigner l'architecture en France. Le Monde. Retrieved from https://www.lemonde.fr/campus/article/2017/06/24/nous-pensons-qu-il-faut-dynamiter-lafacon-d-enseigner-1-architecture-en-france_5150594_4401467.html

Norton, B., Lo, S. N. G., Cronin, K. P., \& Yohanis, Y. G. (1995). Towards the harmonious integration of information technology in building design and construction. International Journal of Ambient Energy, 16(2), 95-109. https://doi.org/10.1080/01430750.1995.9675675

Perisic, A., Lazic, M., \& Perisic, B. (2016). The Extensible Orchestration Framework approach to collaborative design in architectural, urban and construction engineering. Automation in Construction, 71, 210-225. https://doi.org/10.1016/j.autcon.2016.08.005

Reinschmidt, K. F., Griffis, F. H. (Bud), \& Bronner, P. L. (1991). Integration of Engineering, Design, and Construction. Journal of Construction Engineering and Management, 117(4), 756-772. https://doi.org/10.1061/(ASCE)07339364(1991)117:4(756)

Santos, R., Costa, A. A., \& Grilo, A. (2017). Bibliometric analysis and review of Building Information Modelling literature published between 2005 and 2015. Automation in Construction, 80, 118-136. https://doi.org/10.1016/j.autcon.2017.03.005

Sattler, L., Lamouri, S., Pellerin, R., Paviot, T., \& Deneux, D. (2019). A literature review about BIM interoperability and collaboration for design and construction between 2013 and 2018. (Under Revier).

Sattler, L., \& Maigne, T. (2018). L'ère post-BIM - Pour une obsolescence déprogrammée: une étude de cas de deux projets de Frank Gehry en France, de 2008 à 2016. Dnarchitecture. Retrieved from https://dnarchi.fr/experimentations/lere-post-bimpour-une-obsolescence-deprogrammee-une-etude-de-cas-de-deux-projets-de-frank-gehryen-france-de-2008-a-2016-i-iii/

van Nederveen, G. A., \& Tolman, F. P. (1992). Modelling multiple views on buildings. Automation in Construction, 1(3), 215-224. https://doi.org/10.1016/0926-5805(92)90014-B

Yamazaki, Y.(1992). Integrated design and construction planning system for Computer Integrated Construction. 6. 